\title{
The Distribution of Supernova Remnants in the Galaxy
}

\author{
By D. A. GREEN \\ Mullard Radio Astronomy Observatory, Cavendish Laboratory, Madingley Road, Cambridge \\ CB3 OHE, UNITED KINGDOM
}

Observational selection effects and the lack of accurate distances for most Galactic SNRs pose problems for studies of the distribution of SNRs in the Galaxy. However, by comparing the observed Galactic longitude distribution of high surface brightness SNRs with that expected from simple models - which avoids some of the problems with selection effects and the lack of distances - a Gaussian scale length of $\approx 7 \mathrm{kpc}$ in Galactocentric radius is obtained for SNRs.

\section{Introduction}

The distribution of SNRs in the Galaxy is of interest for many astrophysical studies, particularly in relation to their energy input into the ISM and for comparison with the distributions of possible progenitor populations. Such studies are, however, not straightforward. First, current catalogues of SNRs miss objects due to observational selection effects. Second, there are no reliable distance estimates available for most identified remnants. Here I use a sample of 182 Galactic SNRs from a recently revised catalogue (this proceedings), all but one of which have observed radio flux densities and angular sizes, to derive the distribution of SNRs in the Galaxy by comparing the observed distribution of bright remnants with Galactic longitude with that expected from simple models.

\section{The Problems}

\subsection{The Selection Effects}

Although, as discussed by Aschenbach (this proceedings), many new SNRs may soon be identified from the ROSAT X-ray survey, the identification of SNRs in existing catalogues has, generally, been made at radio wavelengths. Such identifications are, basically, limited by two selection effects: (i) the surface brightness of the remnant must be above the sensitivity limit of the observations and be readily distinguishable from the Galactic background emission, and (ii) the angular size of the remnant must be at least several times the resolution of the observations (these selection effects are discussed in detail in Green 1991). It is thought that current catalogues are nearly complete in the whole Galactic plane down to a surface brightness at $1 \mathrm{GHz}, \Sigma_{1 \mathrm{GHz}}$, of $\approx 8 \times 10^{-21} \mathrm{~W} \mathrm{~m}^{-2} \mathrm{~Hz}^{-1} \mathrm{sr}^{-1}$. The lower limit for completeness for angular size is $\approx 8$ arcmin. This is illustrated in Fig. 1, which shows histograms of the surface brightnesses and angular sizes of catalogued SNRs.

These effects mean that not only are old faint remnants missing from current catalogues, but there is also a deficit of young but distant SNRs. It will be difficult to make catalogues of Galactic remnants complete to much lower surface brightnesses, as some regions of the Galactic plane - such as the Galactic center, and Cygnus - have very complex emission on a wide variety of angular scales. Such "confusion" can, to some extent, be avoided by using multi-frequency or polarization radio observations, or 
a)

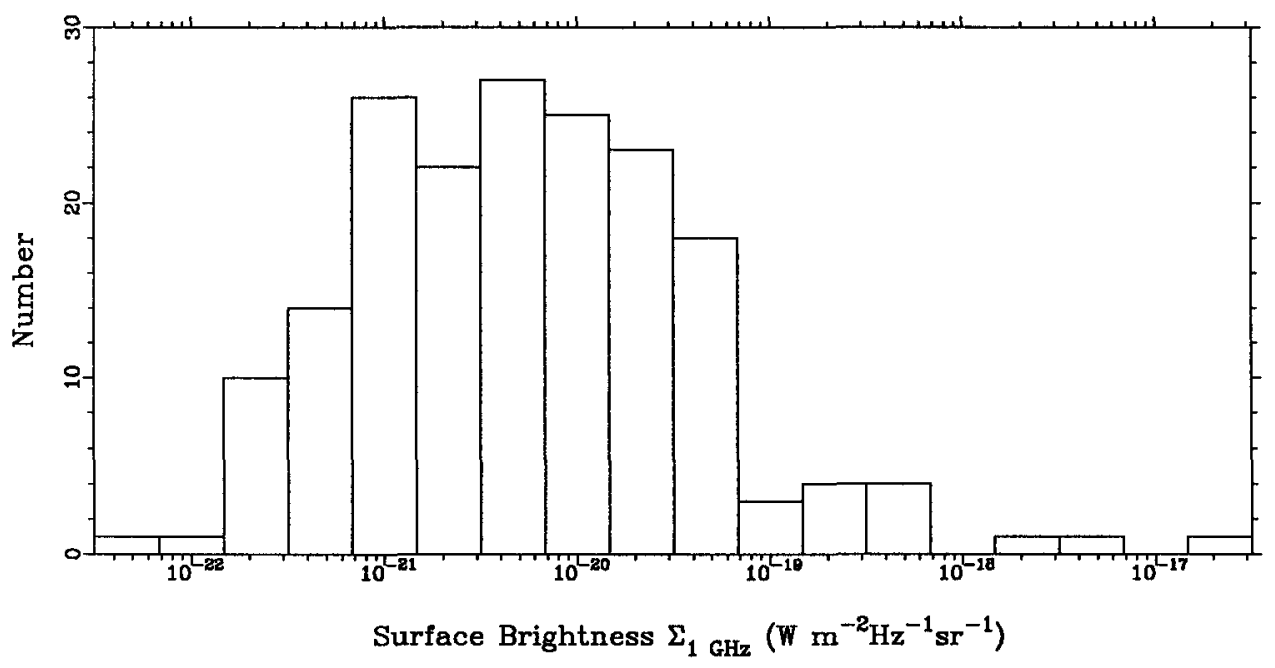

b)

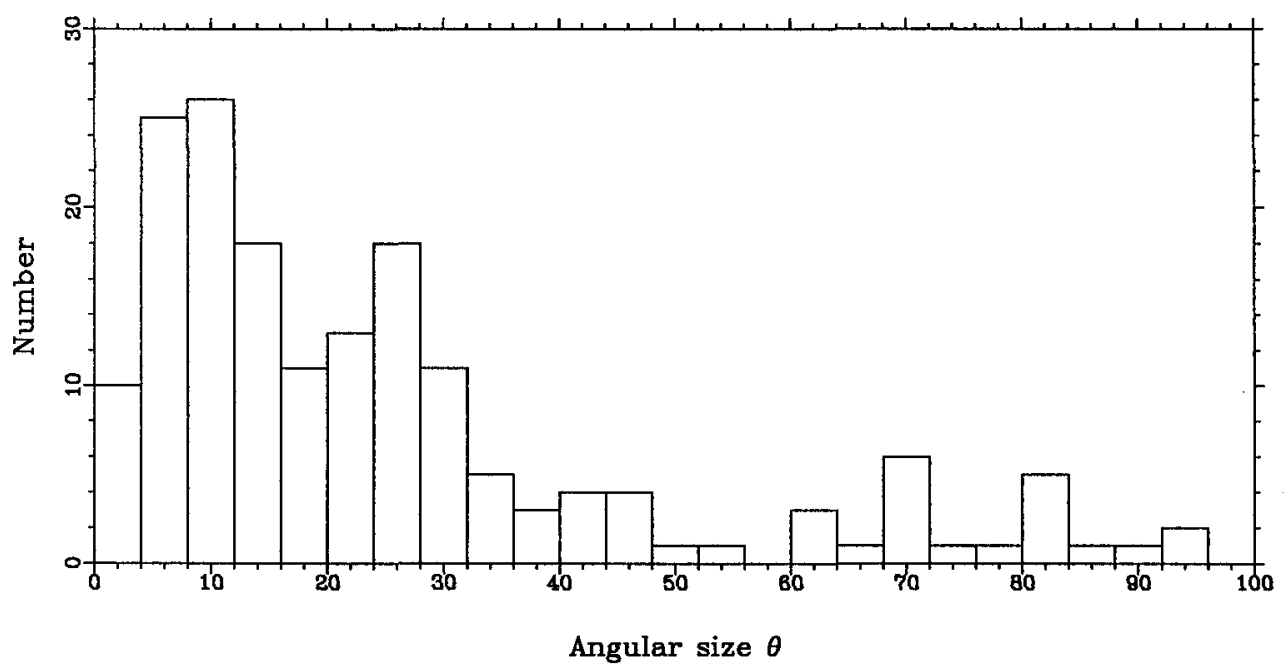

FIGURE 1. Histograms of (a) surface-brightness and (b) angular size, for catalogued SNRs (10 large angular diameter remnants do not fit on this plot). The nominal completeness limits in surface-brightness and angular size are $\approx 8 \times 10^{-21} \mathrm{~W} \mathrm{~m}^{-2} \mathrm{~Hz}^{-1} \mathrm{sr}^{-1}$ and $\approx 8$ arcmin respectively.

comparisons with infrared and X-ray surveys, to separate out different components of emission. However, the problem of identifying faint SNRs in the complex regions of the Galactic plane is difficult.

There have been several searches for the missing small SNRs (Green \& Gull 1984; Reich et al. 1985; Green 1985, 1989; Helfand et al. 1989; Sramek et al. 1992), but with little success. This is largely due to the large number of compact radio sources in the Galactic plane, most of which are extragalactic. For example, the Effelsberg $2.7-\mathrm{GHz}$ plane survey (of the region $358^{\circ}<l<240^{\circ},|b|<5^{\circ}$, with a beam of 4.6 arcmin) contains 6495 small-diameter sources less than 12 arcmin in apparent size (Fürst et al. 1990a), and most of these have not been observed in detail. (It is, however, implausible 


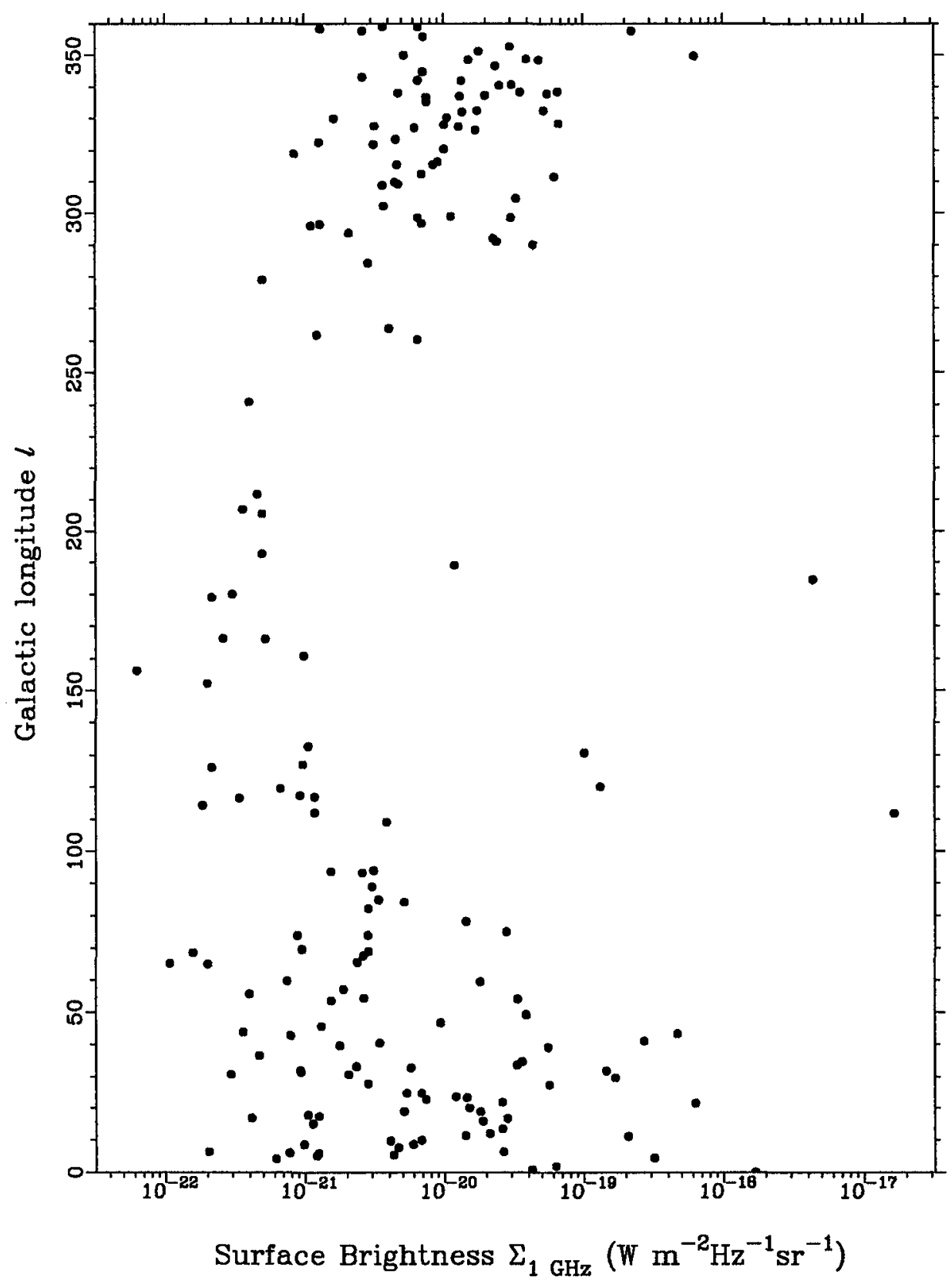

FIGURE 2. Plot of Galactic longitude against surface brightness for catalogued SNRs. Note that there are relatively many more faint remnants in the 2nd and 3rd quadrants $-90^{\circ}<l<270^{\circ}$ - than in the 1st and 4th quadrants.

that any very high surface brightness remnants like Cas A or the Crab nebula have been missed - see Green 1985.)

Although many SNRs have been identified with surface brightnesses less than limit quoted above, these are predominantly in regions of low background Galactic radio emission, such as high Galactic latitudes, or in the 2nd and 3rd Galactic quadrants (the 


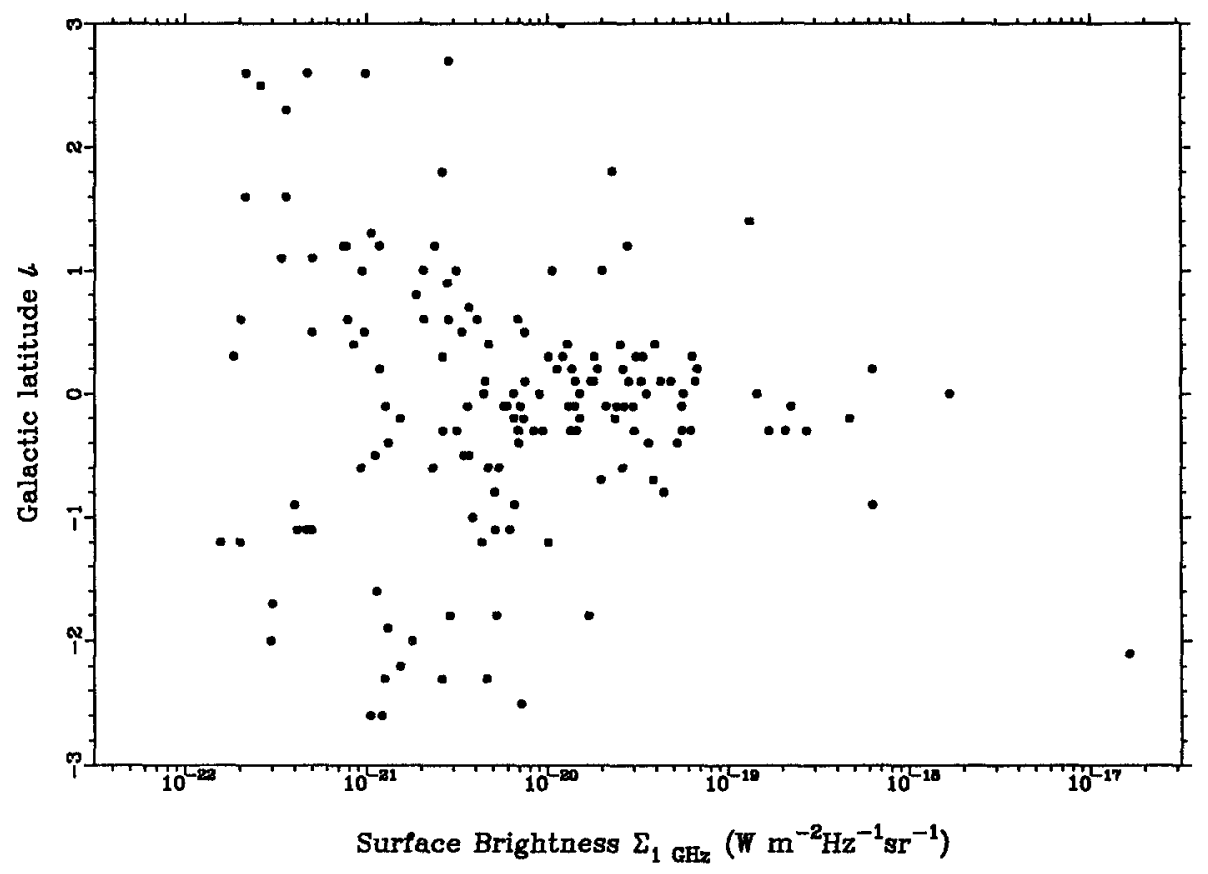

FIGURE 3. Plot of Galactic latitude against surface brightness for catalogued SNRs (25 remnants with $|b|>3^{\circ}$ are not included on the plot). Note that there are relatively many more faint remnants away from $b=0^{\circ}$.

Galactic "anticenter" region). This is illustrated in Figs 2 and 3, which clearly show that there are relatively more faint remnants in the anticenter region or away from $b=0^{\circ}$ where background confusion is less of a problem. (Note also that more fainter SNRs are identified in the 1st quadrant, compared with the 4 th, because the Effelsberg $2.7-\mathrm{GHz}$ survey - Reich et al. 1990 and Fürst et al. 1990b - cover these regions). Since all SNRs in the anticenter quadrants are outside the Solar Circle at large Galactocentric radius, it is difficult to disentangle this bias from any intrinsic variation in the properties of SNRs with Galactic coordinates. This selection effect accounts for the very broad distribution of SNRs derived by Li et al. (1990), who included all SNRs in their analyses, and were therefore biased to distributions showing an excess at large Galactocentric radii.

Van den Bergh $(1988 a, b)$ discussed the distribution of observed SNRs and noted that high surface brightness remnants (in this case taken to be $\Sigma_{1 \mathrm{GHz}}>3 \times 10^{-21} \mathrm{~W}$ $\mathrm{m}^{-2} \mathrm{~Hz}^{-1} \mathrm{sr}^{-1}$ ) are concentrated in a thin nuclear disk. As noted by Fürst's comments to van den Bergh 1988b, this conclusion is strengthened by a more realistic surface brightness completeness limit (see Green 1991).

\subsection{The Distances to SNRs}

The fact that there are no high surface brightness SNRs with large diameters has often been expressed in terms of a " $\Sigma-D$ " relation (usually of the form $\Sigma \propto D^{-n}$, relating surface brightness, $\Sigma$, to linear diameter, $D$ ). If such a relation can be calibrated with remnants at known distances, then diameters and hence distances can be derived for any SNR from its observed surface brightness. However, the correlation in the $\Sigma-D$ plane is poor (Green 1984; Berkhuijsen 1986), and the full extent of the range of properties of Galactic SNRs in the $\Sigma-D$ plane is not currently known, due to the selection effects described above (see also Green 1991). 


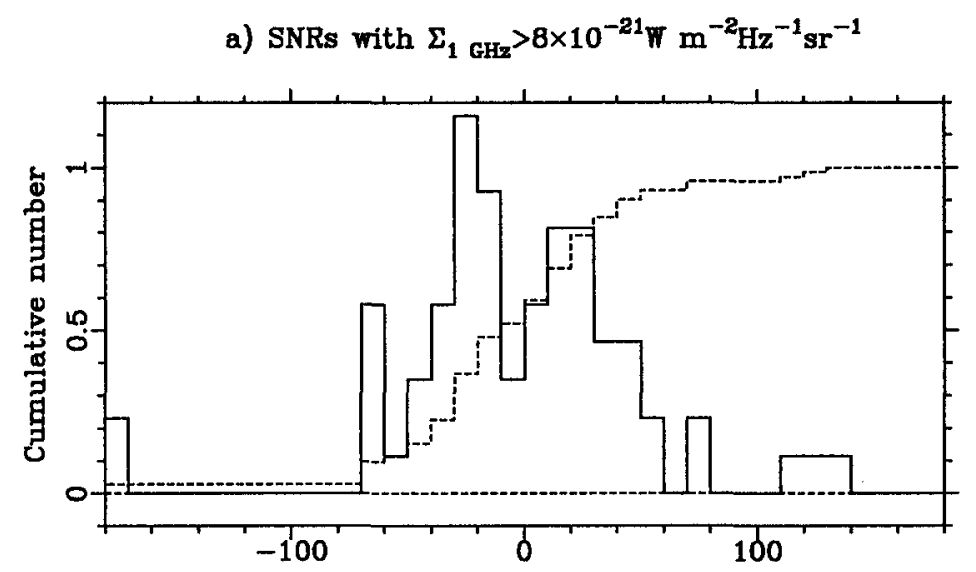

b) all SNRs

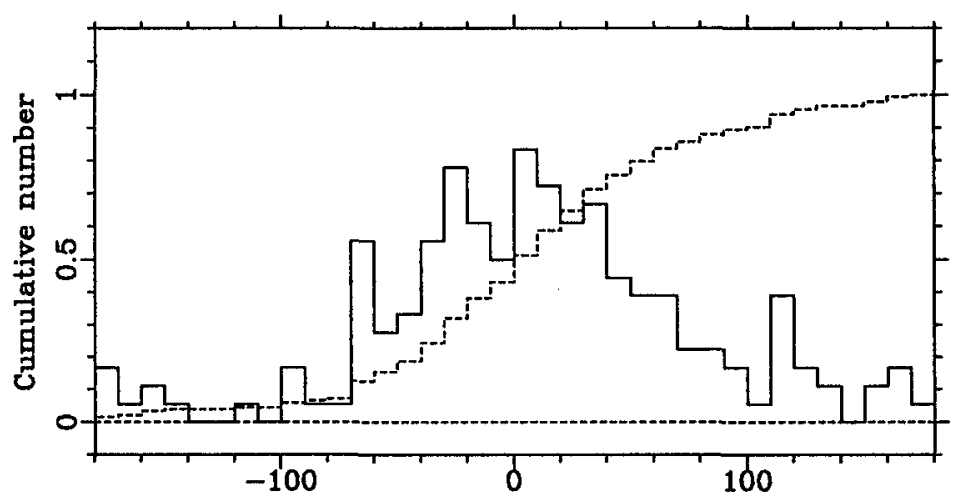

FIGURE 4. Histograms of the distributions of SNRs with Galactic longitude (solid lines) and cumulative distributions (dashed lines) for, (a) high surface brightness remnants (71 with $\left.\Sigma_{1 \mathrm{GHz}}>8 \times 10^{-21} \mathrm{~W} \mathrm{~m}^{-2} \mathrm{~Hz}^{-1} \mathrm{sr}^{-1}\right)$, and (b) all SNRs.

Consequently only an upper limit to the diameter, and hence to the distance, of an SNR can be deduced with any confidence from a given surface brightness (see Berkhuijsen 1986). This boundary in the $\Sigma-D$ plane is not the result of any observational selection effects, and represents some intrinsic limit to the luminosity of radio emission from SNRs, although it does not necessarily represent the evolutionary path of any individual SNR in the $\Sigma-D$ plane. $\Sigma-D$ studies are only made because $\Sigma$ is a distance-independent observational parameter. Much of the correlation in $\Sigma-D$ is due to an intrinsic " $1 / D^{2}$ " bias in this representation compared with the $L-D$ plane (i.e. for a remnant of luminosity $L$, at a distance $d, L \propto S d^{2}$, whereas $\Sigma \propto S / \theta^{2}$, so $\Sigma \propto L /(\theta d)^{2}$, or $\left.\Sigma \propto L / D^{2}\right)$

\section{The Distribution of 'SNRs in the Galaxy}

The problems caused by the $\Sigma$-selection effect on statistical studies can be reduced if such studies are restricted to relatively bright remnants, for which current catalogues are thought to be complete (although some high surface brightness remnants with small angular diameters will still be missing). The distribution of remnants perpendicular to the Galactic plane is obviously affected by the surface brightness selection effect, as faint 
a) $\sigma=5 \mathrm{kpc}$

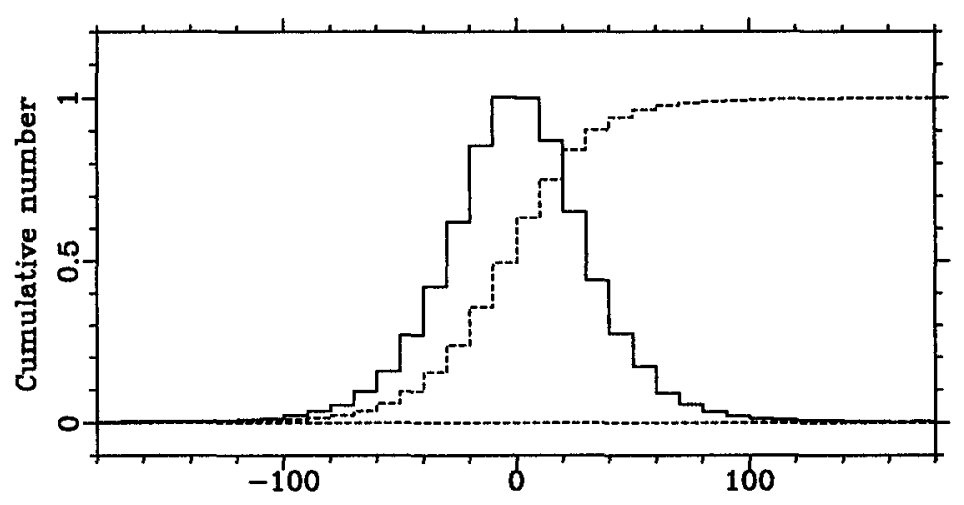

b) $\sigma=7 \mathrm{kpc}$

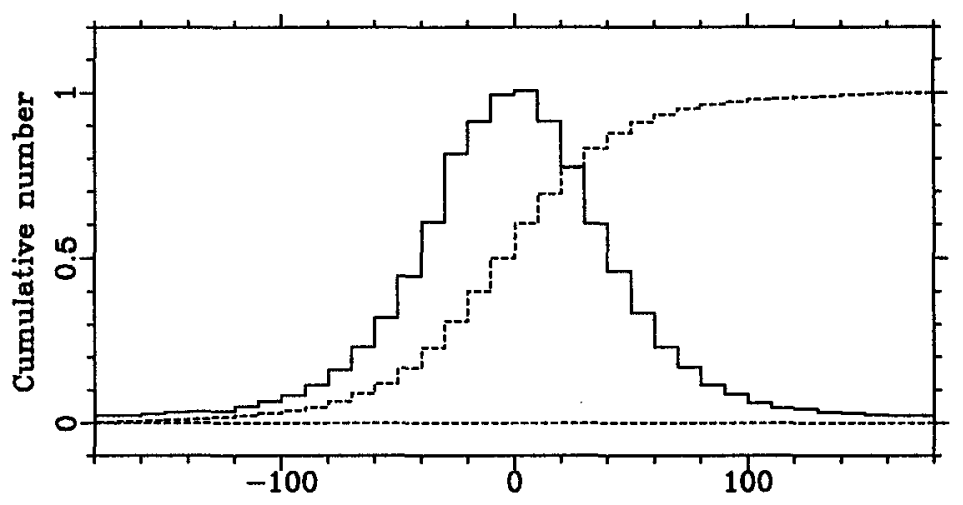

c) $\sigma=8 \mathrm{kpc}$

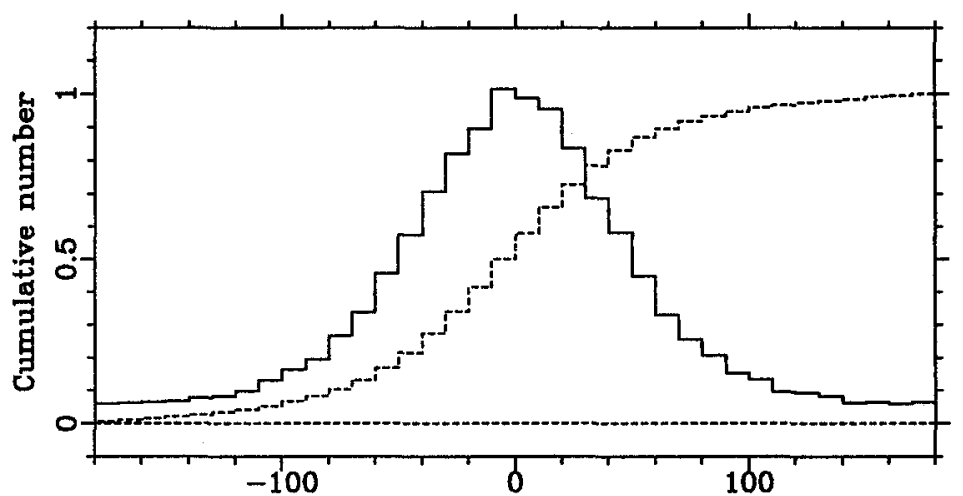

FIGURE 5. Histograms of model distributions of SNRs with Galactic longitude (solid lines) and cumulative distributions (dashed lines) for various Gaussian Galactocentric scale lengths (see text). 
remnants are more difficult to identify near $b=0^{\circ}$. The distribution perpendicular to the plane is further complicated by the warp of the Galaxy outside the Solar Circle. There are indeed more SNRs identified at large positive values of $b$ in the 1st and 2nd quadrants than elsewhere, as is expected because of the warp of the Galactic plane in these directions. In the subsequent discussion I will not address the question of the distribution of SNRs perpendicular to the plane, but concentrate on constraining the distribution in the disk of the Galaxy. To do this, I use the method of $\mathrm{Li}$ et al. - to compare the observed distribution of SNRs with Galactic longitude with that expected from various models. This avoids the problem that we lack accurate distances to individual SNRs.

Fig. 4 shows the observed distribution with Galactic longitude of (a) high surface brightness remnants (71 SNRs in the catalogue with $\Sigma_{1 \mathrm{GHz}}>8 \times 10^{-21} \mathrm{~W} \mathrm{~m}^{-2} \mathrm{~Hz}^{-1} \mathrm{sr}^{-1}$ ), and also (b) for all remnants. This clearly shows that there are relatively many faint remnants in the Galactic anticenter. This also shows evidence for a deficit of SNRs near $l=0^{\circ}$. This may be a true deficit, as might be expected for a decrease in the space density of SN progenitors towards the Galactic center. However, it may also be, in part at least, due to the difficulty of finding remnants in this region of the Galactic plane, due to the very complex background emission. Any remaining incompleteness in current catalogues, both for the surface brightness and angular diameter selection effects, are expected to be worse closer to $b=0^{\circ}$ (because of the increased confusion in the case of the surface brightness selection effect, and the longer line-of-sight through the Galaxy for missing small, i.e. young but distant remnants). Thus, the true distribution in $l$ is likely to be somewhat narrower than is indicated in Fig. 4(a). For comparison with the observed distributions in Galactic longitude I have made simple Monte Carlo models of the distribution of SNRs in the disk of the Galaxy, assuming a simple Gaussian distribution, where the probability distribution varies with Galactocentric radius, $R$, as

$$
\propto \mathrm{e}^{-(R / \sigma)^{2}}
$$

(where $\sigma$ is the Gaussian Galactocentric scale length, assuming the distance to the Galactic Center of $10 \mathrm{kpc}$ ). Fig. 5 shows plots of the expected distribution of SNRs in Galactic longitude of three such models for different scale lengths. Simple comparisons of the observed and model cumulative distributions indicate a scale length of $\approx 7 \mathrm{kpc}$ for the high brightness SNRs. (The much broader distribution in Galactic longitude of all catalogued remnants - due to the selection effects - gives a much larger apparent scale length of $\approx 10 \mathrm{kpc}, \mathrm{cf}$. Li et al.). As noted above, the true distribution is likely to be somewhat narrower than that derived from the observations, due to residual selection effects, so that this scale length is an upper limit.

The distribution of SNRs derived above should, however, be interpreted cautiously, especially when making direct comparisons of it with distributions of other Galactic populations. It is far from clear that the observed SNR distribution actually represents the parent supernovae distribution, as the factors that affect the brightness and lifetime of appreciable radio emission from SNRs are not well understood. The distribution of SNRs could simply reflect the distribution of, say density of the ISM, or magnetic field, if they are the crucial factors in determining the brightness and lifetime of radio emission from SNRs.

\section{REFERENCES}

Berkhuijsen, E. M., 1986, A\&A, 166, 257

Fürst, E., Reich, W., Reich, P., Reif, K., 1990a, A\&AS, 85, 805

Fürst, E., Reich, W., Reich, P., Reif, K., 1990b, A\&AS, 85, 691 
Green D. A., 1984, MNRAS, 209, 449

Green D. A., 1985, MNRAS, 216, 691

Green D. A., 1989, AJ, 98, 1358

Green D. A., 1991, PASP, 103, 209

Green D. A., Gull S. F., 1984, Nature, 312, 527

Helfand, D., Velusamy, T., Becker, R. H., Lockman, F. J., 1989, ApJ, 341, 151

Li, Z.-W., Wheeler, J. C., Bash, F. N., Jefferys, W. H., 1991, ApJ, 378, 93

Sramek, R. A., Cowan, J. J., Roberts, D. A., Goss, W. M., Ekers, R. D., 1992, AJ, 104, 705

Reich, W., Fürst, E., Altenhoff, W. J., Reich, P., Junkes, N., 1985, A\&A 151 L10

Reich, W., Fürst, E., Reich, P., Reif, K., 1990, A\&AS, 85, 633

van den Bergh, S., 1988a, PASP, 100, 205

van den Bergh, S., 1988b, in Kundt, W., ed., Supernova Shells and Their Birth Events, SpringerVerlag, p. 44 